\title{
Research and design of grid connection and waste heat utilization of marine generating units
}

\author{
Anning $\mathrm{Yi}^{1 *}$, Hongtao Guo ${ }^{1}$ \\ ${ }^{1}$ School energy and power engineering, Wuhan university of technology, Wuhan, Hubei, 430063, China
}

\begin{abstract}
This work is based on the use of waste heat from the temperature difference semiconductor heat exchanger, which can effectively use the waste heat in the exhaust gas, and convert it into electrical energy output through the temperature difference semiconductor material, which can increase engine efficiency and reduce energy consumption; at the same time, it can reduce engine noise and vibration. Extended service life. Due to the strong electromagnetic interference and severe vibration of the generator, there are few remote control devices on the market for the generator. This project uses a $2.4 \mathrm{G}$ wireless communication module to control the frequency conversion and speed regulation of the generator. In order to save manpower, start remotely, stop as soon as possible, monitor the operating status of the waste heat temperature difference power generation, reasonably replace the power, start quickly, and reach the electromechanical Integrated product. The realization of intelligent frequency conversion technology can adjust the engine speed according to different electrical appliances, adapt to external loads, realize automatic voltage adjustment, and save fuel consumption. The grid-connected system solves the frequency and phase problems of generators of different models, generations, and manufacturers in parallel, and realizes the re-mixing of old generators, which greatly improves the service life of engines and the best power generation supply, and reduces power generation systems and storage. The configuration cost of the energy unit improves the comprehensive utilization rate of the equipment, has a higher working efficiency, has good economic benefits, and can achieve the purpose of energy saving and emission reduction.
\end{abstract}

\section{Introduction}

At present, most ship generators have low power generation efficiency, high noise, and exhaust pollution of the environment, which cannot achieve efficient applications. Various types of engines on the market are uneven. As the power consumption continues to increase, the number of units is also increasing, and the generators have a long service life. There are many generators of different sizes and models that form a single unit. For chip power supply, because the load is not easy to deploy, it causes large frequency and voltage fluctuations, which affects the quality of power supply. At the same time, the output power of the generator is not fully utilized, which reduces the efficiency and increases the fuel consumption per kilowatt-hour. The current generator is relatively noisy and the exhaust gas is polluted heavily. Every time the generator is turned on, people need to operate the interface, pull the start handle and adjust the size of the damper. The operation is tedious, and the shutdown is very inconvenient and difficult. Learned the operating conditions of the generator in the working process. Once the working conditions suddenly change, the voltage suddenly changes, and the power frequency changes. If the generator is not adjusted in time, it will bring serious consequences to the load circuit and damage the equipment. ${ }^{[1]}$
At present, these solutions on the market are to change the current operation of multiple motors to multiple parallel operation. At the same time, the grid connection increases the load capacity of the power grid, which determines the stable voltage and frequency. However, it is more difficult for multiple units to run in parallel, and it is easier to run the same model in parallel. In fact, most units' generators are temporarily purchased as the power consumption increases, resulting in different models and mixed use of old and new products. Difficulty has become the reason why many units still stand alone.Gasoline generator fuel only accounts for about $40 \%$ of the energy consumed. The remaining $60 \%$ of the energy is not fully utilized. Of this energy, $30 \%-45 \%$ of the energy is taken away by engine exhaust emissions. Most of this energy is dissipated through heat, causing serious air pollution. In addition, the gasoline generator has a high exhaust pressure and high temperature, and the exhaust temperature can reach about $400^{\circ} \mathrm{C}$.

Based on the above background, the project team designed the marine grid-connected engine unit based on the waste heat utilization of the temperature difference semiconductor heat exchanger. Compared with the current market engine units, this device uses the waste heat for temperature difference power generation, which not only saves energy, but also Because the heat is used for temperature difference power generation, the thermal

${ }^{*}$ Corresponding author's e-mail: 981282100@qq.com 
efficiency of the gas turbine is improved and the noise and vibration of the ship are reduced to a certain extent. ${ }^{[2]}$

\section{Proposed study plan}

\subsection{Temperature difference power generation module}

The temperature difference power generation module used in this device has four layers, and the connection topology of each layer adopts the connection mode of parallel connection of modules in columns, serial connection of modules between columns, and serial connection of modules between layers. First, the exhaust gas of the generator is equipped with a temperature difference semiconductor heat exchanger device. The structure of the heat exchanger is laminated and stacked by multiple single-layer temperature difference power generation devices. The structure is compact, including multiple waste heat channels and cooling channels, and the total working area Large, exhaust gas heat energy utilization efficiency is high. The cascading structure can add / remove channels, realize flexible configuration, and meet various power requirements.

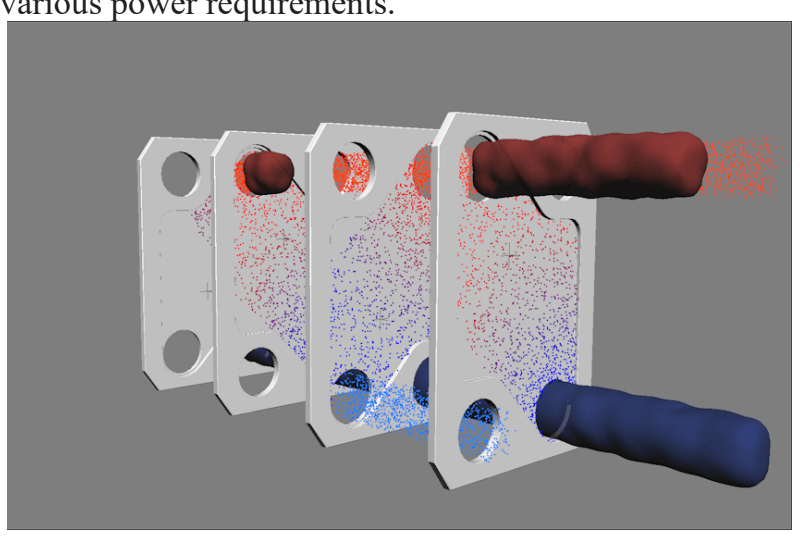

Figure 1. Working principle of temperature difference semiconductor

\subsection{Gasoline generator grid-connected conversion device}

\section{(1) Control module}

Genset parallel controller is used for the manual / automatic parallel system of multiple gensets with the same capacity or different capacities, and is suitable for the constant power output of a single genset and the grid connection to the mains to achieve automatic startup / stop / parallel operation, data Measurement and alarm protection.

(2) Parallel control system

The controller obtains the target voltage value and the target current ratio from the host controller; the generator is driven by the motor to generate three-phase $\mathrm{AC}$ power and rectify it in parallel to supply power to the load. When the system is running, the controller receives the output current signal of the generator from the current sensor and collects the output voltage signal. The control amount is calculated by the specific control strategy. The excitation current of the two generators is controlled by the pulse width modulation signal (PWM)., And then control the voltage and target current ratio output of the system.

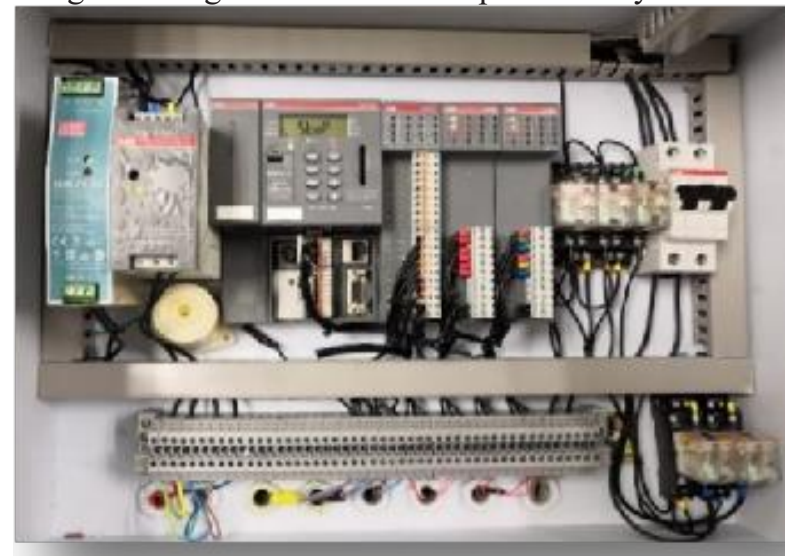

Figure 2. Control module diagram

(3) Voltage automatic frequency converter

At present, generators on the market have popularized AVR (Automatic Voltage Regulator) components, which can automatically adjust the generator's speed and throttle according to the generator load. No manual intervention is required to effectively reduce the impact of the starting surge current on the power grid. The additional strong excitation provides support when the grid fault voltage drops, and strengthens the grid stability. Additional adjustment to achieve reasonable distribution of reactive power.

\section{Research content and research objectives of the project}

Design an engine unit based on the exhaust gas waste heat utilization of a temperature difference semiconductor heat exchanger, which can effectively utilize the waste heat in the exhaust gas, convert it into electrical energy output through the temperature difference semiconductor material, increase engine efficiency, reduce energy consumption, and reduce engine noise And vibration to extend service life. This project uses a $2.4 \mathrm{G}$ wireless communication module to control the frequency conversion and speed regulation of the generator, save manpower, start remotely, stop on demand, start quickly, and achieve mechatronics. This project uses intelligent frequency conversion technology, which can adjust the engine speed according to different electrical appliances, adapt to external loads, realize automatic voltage adjustment, and save fuel consumption. The gridconnected system solves the frequency and phase problems of generators of different models, generations, and manufacturers in parallel, and realizes the re-mixing of old generators, which greatly improves the service life of engines and the best power generation supply, and reduces power generation systems and storage. ${ }^{[3]}$ 


\section{Innovative and advanced}

1) The use of exhaust gas waste heat from temperature difference semiconductor heat exchangers can efficiently save energy, reduce the configuration costs of power generation systems and energy storage units, increase the overall utilization of equipment, and achieve energy conservation and emission reduction;

2) Utilize remote control and intelligent frequency conversion, adapt to external load, realize automatic voltage adjustment, save fuel consumption;

3) The grid-connected system realizes the mixed use of old generators, which greatly improves the service life of the engine and the power supply.

\section{Expected economic benefits and promotional value}

In this project, the temperature difference between the exhaust heat of the gasoline generator and the cooling water is used to generate electricity. ${ }^{[4]}$ A stacked temperature difference semiconductor heat exchanger is installed. The cold end is continuously cooled by the external circulating water pump, and the temperature difference is obtained. The obtained electricity is stored in In the battery, according to different working conditions, the corresponding electric energy output is replaced for work. Based on the flat-type temperature difference power generation device, there are two layers of hot-end water tanks, three layers of cold-end water tanks, and four-layer temperature difference power generation modules. ${ }^{[5]}$

After that, we designed a temperature difference semiconductor experimental bench according to the characteristics of the generator's operating conditions, and carried out the relationship between the output voltage and power of each temperature difference power generation device under the condition of forced water cooling. The experiment is performed between the hot end temperature, the cold end temperature, and the cold and hot end temperature. ${ }^{[6]}$ The flue gas temperature is $400^{\circ} \mathrm{C}$, which can output $427.23 \mathrm{~W}$ thermal efficiency of $5.01 \%$, it can output $241.244 \mathrm{~W}$ thermal efficiency $2.7 \%$. Through actual measurement, the improved device can achieve: singlemachine energy saving of $14.75 \%$, and generator set energy saving of $21.34 \%$. Combined with remote control to monitor the real-time condition of the engine, and using structural innovations, the excess energy utilization rate reached $20 \%{ }^{[7]}$.

\section{Conclusion}

This project is based on the most critical energy utilization issues in energy saving and emission reduction. The products designed can fully reduce the labor cost consumption. The temperature difference semiconductor heat exchanger can improve the efficiency of generators, reduce exhaust emissions, and increase the number of grid-connected systems. The two generators generate electricity together, increase the output power, and use the generator set under different conditions to run. Both the grid-connected system and temperature difference semiconductor power generation require a set of remote intelligent control to monitor the real-time condition of the generator. Therefore, the project has good prospects and broad development space.

\section{References}

1. Li Ru. Discussion on Several Issues of Parallel Operation of Small Diesel Generators. 1999.5

2. PATELS. Utilization and development of marine energy [J]. Shanghai Electric Power, 2009 (1): 32-38

3. Liu Shengguan, Xie Dian, et al. Marine wave, wind and light integrated power ship: China, 201310462964.3[P]. 2013-10-08.

4. Yuan, X.H. Research on thermoelectric conversion technology of automobile engine exhaust heat temperature difference power generation device. $\mathrm{PhD}$ dissertation. 2012, 2.

5. Lin Shigao, Liu Xiaolin. The manipulator attitude smoothing algorithm based on the fifth-order polynomial [J]-Manufacturing Automation 2013(21).

6. Wang, X. Z.; He, Y. R.; Liu, X.; Zhu, J. Q. Enhanced direct steam generation via a bio-inspired solar heating method using carbon nanotube films. Powder Technology. 2017, 321, 276-285.

7. Elimelech, M.; Phillip, W. A. The Future of Seawater Desalination: Energy, Technology, and the Environment. Science 2011, 333, 712-717. 